\title{
Genetic evaluation of gestation length and its use in managing calving patterns
}

\author{
M. Haile-Mariam ${ }^{1 *}$ and J. E. Pryce ${ }^{1,2}$ \\ ${ }^{1}$ Agriculture Victoria, AgriBio, Centre for AgriBioscience, Bundoora, Victoria 3083, Australia \\ ${ }^{2}$ School of Applied Systems Biology, La Trobe University, Bundoora, Victoria 3083, Australia
}

\begin{abstract}
Data collected from Australian Holstein cows that calved between 1995 and 2016 were used for estimating genetic parameters and genetic evaluation of gestation length (GL). Genetic parameters were estimated using a sire maternal grandsire model considering GL in heifers as correlated, but a different trait to adult cows and animal repeatability model. The key objective of this study was to assess if selective mating of bulls with short GL estimated breeding values (EBV) can help to modify calving patterns in predominantly pasturebased production systems and thereby contribute to the reduction of calving induction. The mean GL in heifers and adult cows was 280 and 281 d, respectively. The heritability of direct GL was 0.28 in heifers, which is slightly lower than in adult cows (0.36). The maternal heritability of GL was close to 0.04 in both heifer and adult cows. The genetic correlation between direct effects in heifers and adult cows was lower (0.88) than between maternal effects (0.94). A genetic evaluation model that considered heifer and adult cow data as the same trait in a repeatability animal model showed adequate variability in EBV for both direct and maternal GL. The genetic trend in direct GL EBV declined from 2005 in bulls and from 2006 in cows. Selective mating of bulls with short direct GL EBV showed that the GL and calving interval of their mates can be modified by up to $3.5 \mathrm{~d}$ for GL and $2.0 \mathrm{~d}$ for calving interval. Relative to parent average, the use of genotype data to calculate genomic EBV increased the reliability of EBV by up to $11 \%$ in validation bulls when daughter trait deviation of bulls with trait deviation of cows (11\%) and deregressed breeding values (8\%) were used as response variables. On average, for animals with only genotype data, the GL EBV can be predicted with about 50 to $60 \%$ reliability (expected) depending on the response
\end{abstract}

Received April 26, 2018.

Accepted September 4, 2018.

*Corresponding author: Mekonnen.HaileMariam@ecodev.vic.gov.au variable (deregressed breeding values or daughter trait deviation and trait deviation) and the size of reference set. Overall, the results of this study show that calving patterns can be made tighter by selectively mating short GL EBV bulls to cows that do not become pregnant early in the mating season. Additionally, better reproductive management and the use of bulls with high female fertility EBV are still crucial for the success of a pasture-based dairy production system.

Key words: gestation length, direct genetic effect, estimated breeding values, seasonal calving system

\section{INTRODUCTION}

Gestation length (GL) is largely influenced by the genes that the calf inherits from its sire (direct) with a heritability $\left(\mathrm{h}^{2}\right)$ of about 0.40 . These moderate $(0.30)$ to high (0.50) direct $\mathrm{h}^{2}$ estimates (Winkelman and Spelman, 2001; Hansen et al., 2004; McClintock 2004; Norman et al., 2009; Eaglen et al., 2013) suggest that genetic progress for this trait could be made. On the other hand, the $\mathrm{h}^{2}$ of the maternal effect is low (0.05 to $0.10)$ and progress on this component of GL is expected to be slow.

A study using US data showed strong evidence that choice of service bulls (direct GL) can either increase or decrease the GL of cows to which they are mated, which means herd managers could use EBV of GL to aid in the management of calving schedules or to help in predicting calving dates (Norman et al., 2011). Norman et al. (2011) also found that an intermediate GL was optimal for productive life, calving ease, stillbirth, culling, and days open. Other studies, such as Hansen et al. (2004) using Danish Holstein data and Eaglen et al. (2013) using UK Holstein data, also did not support selection for either decreased or increased GL. On the other hand, Jenkins et al. (2016), using data from New Zealand dairy herds, concluded that overall the net effect of shortened GL is likely to be economically positive.

In the seasonal calving systems that dominate New Zealand dairying, genetic evaluations and selection for 
direct GL have occurred for several years (Winkelman and Spelman, 2001). The New Zealand breeding company Livestock Improvement Corporation (LIC) have selectively bred for short-GL Holstein Friesian bulls, which has reduced GL EBV by $0.094 \mathrm{~d}$ per annum in recent years (Greg Marshall, Development Analyst Lead, LIC, Hamilton, New Zealand; personal communication). The LIC market bulls can shorten GL by up to $10 \mathrm{~d}$ (LIC, 2018). In Australian herds that practice seasonal or split calving, considerable interest also exists in identifying bulls whose calves are born after a shorter pregnancy relative to the average bull. Reducing the GL of progeny of cows that conceive late in the mating season has been identified as a way of reducing the need for artificial induction of parturition (Dairy Australia, 2018b), which could also lead to more time to recover before the next $\mathrm{AI}$ and as a means of maintaining a 365-d calving interval. However, the availability of data on GL from Australian herds has been limited, so genetic evaluations have not been carried out until now.

Data on GL has been accumulating since the mid1990s and there is now an opportunity to carry out genetic evaluations and publish EBV for GL (McClintock, 2004). If enough range exists in direct GL EBV of bulls, short-GL EBV bulls can be selectively mated to cows that did not become pregnant before the end of the mating season. In addition to calculating EBV for GL and quantifying the range, a need exists to demonstrate the benefits of the use of short-GL EBV bulls on important traits, such as the calving interval (CInt) of their mates.

In Australia, producing EBV for GL may help the effort to improve calving patterns by selectively mating short-GL EBV bulls to cows that are not pregnant toward the end of the mating season. The current practice is that bulls used for mating at the end of the mating season are often natural service bulls, which may not have enough GL data for estimation of reliable EBV. In addition, most Interbull member countries currently do not have genetic evaluations for GL (Interbull, 2018), so overseas bulls are less likely to have GL EBV. However, both natural service and overseas bulls can be genotyped, so developing a genomic evaluation may be useful to obtain EBV for overseas and natural service bulls.

The main aim of our study was to develop genetic evaluations for GL and assess its effect on CInt of the cows that are mated to bulls with different GL EBV. Here we (1) compared estimates of genetic parameters using different models; (2) calculated EBV for GL and quantify the range in EBV; (3) calculated genomic EBV for GL and compared the increase in reliability of genomic EBV relative to parent average for validation bulls with no progeny; and (4) tested the effectiveness of using GL EBV of bulls on the CInt and GL of their mates.

\section{MATERIALS AND METHODS}

\section{Data}

The data used for our study was extracted from the DataGene database (Melbourne, Australia; formerly known as Australian Dairy Herd Improvement Scheme) and included animals that calved between 1995 and 2016. From these data, Holstein cows that were mated to Holstein bulls and that were confirmed to be pregnant were selected. If the time in days from last recorded mating to calving was between 260 and $295 \mathrm{~d}$, the record was valid. The GL was only calculated if the calving was coded as normal, which means pregnancies that ended in abortion or induction were excluded.

The GL data from DataGene does not include the sex of calf, but sex of calf has a known effect on GL (e.g., Norman et al., 2009). Therefore, information on sex was incorporated by merging the GL data with calving difficulty data and by searching in the pedigree data, where the sire (bull) and dam (cow) and birth date and sex of most animals are registered. A third category of unknown sex was used where the sex of the calf born could not be determined in this way.

The GL data extracted from the database were mostly of adult cows, with only about $11 \%$ of the calvings being from heifers calving for the first time. This is mainly because some Holstein breeders in Australia deliberately mate heifers to non-Holstein bulls to minimize calving difficulty. For example, only $77 \%$ of Holstein heifers were mated to Holstein bulls, whereas in the all-data set about $94 \%$ of Holstein cows were mated to Holstein bulls.

The GL that was extracted from the DataGene database links each GL record with the cow that calved and is therefore suitable for analyses using a sire and maternal grandsire model. However, the data need to be transformed to animal model format, which was achieved by considering each GL of the calf born in addition to the cow calving. This was done by searching for a calf born on the calving date from the cow and the bull (service bull) in the pedigree data used for genetic evaluation by DataGene. If a calf was found, then the data including identification number and sex were added to the GL data file (this strategy was successful for $22 \%$ of the cases, of which $99 \%$ were females). If the expected animals were not in the pedigree file, then the pedigree file was extended to include animals with the same cow and bull (as parents) on the same date of birth. 
To achieve the objectives of the current study, the following data sets were used for evaluation:

(1) GL data of the calving dates of cows (i.e., birth of animals) that happened between January 1995 and December 2012 were used as a reference set for validation of genomic prediction as well as for calculating parent average (i.e., EBV) for validation bulls using pedigree and also direct GL EBV of bulls used for testing effectiveness of the EBV on calving interval of their mates; these data are referred to as early data; and

(2) all GL data (January 1995 to July 2016) was used to carry out genetic evaluations for genetic trend estimation and for obtaining evaluations for bulls used for cross validation; this data set is referred to as all data.

These 2 data sets were used to calculate EBV, daughter trait deviations (DTD), deregressed BV (DBV), and trait deviation (TD) based on the periods. The EBV, DBV, DTD, and TD using the early data were used for genomic predictions and cross-validation. To assess if the GL EBV of bulls affect the CInt of their mates, cows that were known to be mated after 2012 to bulls with EBV calculated on early data and their CInt from the all data set were extracted. Further description of the data set used is provided with the applied statistical methods in the following sections.

For estimating genetic parameters, cows were selected from the all data set described above if they have a known sire and dam and they calved for the first time between 18 and 34 mo of age. The maximum age at first calving was restricted to 34 mo to exclude cows that calved for a second time and did not have a first calving included in the DataGene database. From these, data from the 430 largest herds that supplied over 200 records each were selected. A description of the data used for genetic parameter estimation is shown in Table 1.

Sires of cows (i.e., maternal grandsires of the calves) and mating bulls (sires of the calves) with at least 5 calves with GL records were selected. The herd-yearseason-age groups (contemporary groups) that have at least 2 GL records were then selected. For cows that were selected as above, their subsequent GL records were included to carry out analyses of multiparity genetic parameter estimation. In the data used for estimating genetic parameters, about $63 \%$ of the cows had data on subsequent parities.

\section{Statistical Analyses}

The basic fixed effect model for analyzing data included a contemporary group effect defined using the herd-year-season-age of calving (2 seasons, January to June and July to December, and 2 age groups of animals, those that calved before $30 \mathrm{mo}$ of age and after 30 mo of age), sex of calf (male, female, and unknown), and month of calving. In addition, age at calving in months was fitted as a covariate (linear and quadratic). The random effects fitted varied depending on the models fitted. As the main objective of the analyses is prediction of EBV for direct GL in all the models, calf born or mating bull was fitted as a random effect with relationship. The other additional random effects fitted were the random effect of cow, to account for repeated GL records of cows (when data from multiple parities were considered) and the genetic effect of cow or maternal grandsire effects to assess if maternal effect is important. All analyses were performed using ASReml (Gilmour et al., 2009).

The most complete model used in our study in matrix notation can be represented as:

$$
\mathbf{y}_{\mathrm{T}}=\mathbf{X}_{\mathrm{T}} \mathbf{b}_{\mathrm{T}}+\mathbf{Z}_{\mathrm{T}} \mathbf{s}_{\mathrm{T}}+\mathbf{W}_{\mathrm{T}} \mathbf{m g} \mathbf{s}_{\mathrm{T}}+\mathbf{C}_{2} \mathbf{p} \mathbf{e}_{2}+\mathbf{e}_{\mathrm{T},},[1]
$$

where $\mathbf{y}_{\mathrm{T}}$ is the observation on 2 traits ( $\mathrm{T}$ is trait 1 for heifer GL and T is trait 2 for cow GL), $\mathbf{X}_{\mathrm{T}}$ is the incidence matrix for fixed effects, $\mathbf{b}_{\mathrm{T}}$ is the vector of fixed effects, $\mathbf{Z}_{\mathrm{T}}$ is the incidence matrix for random effects of sire of the calf born, $\mathbf{s}_{\mathrm{T}}$ is the vector of random sire genetic effects, $\mathbf{W}_{\mathrm{T}}$ is the incidence matrix for random

Table 1. Description of the Holstein-Friesian data used for genetic parameter and genetic evaluation of gestation length

\begin{tabular}{|c|c|c|c|c|}
\hline Item & \multicolumn{2}{|c|}{ Data for parameter estimation } & \multicolumn{2}{|c|}{ All data } \\
\hline Gestation length (GL) & $280.2(6.2)^{1}$ & $281.5(5.4)^{1}$ & $280.3(6.3)^{1}$ & $281.9(5.7)^{1}$ \\
\hline No. of herds & 373 & 429 & 1,231 & 3,311 \\
\hline No. of mating bulls (sire) & 1,415 & 4,301 & 2,072 & 8,692 \\
\hline No. of maternal grandsires & 3,747 & 3,747 & 5,078 & 14,338 \\
\hline
\end{tabular}

${ }^{1}$ Standard deviation of GL.

${ }^{2}$ Value in parenthesis is number of cows. 
maternal grandsire effect of the calf, $\mathbf{m g s}_{\mathrm{T}}$ is the vector of random maternal grandsire genetic effects of the calf, $\mathbf{C}_{2}$ is the incidence matrix for the random permanent environmental effects of cow fitted to cow GL only, $\mathbf{p e}_{2}$ is the vector of random permanent environment of cow, and $\mathbf{e}_{\mathrm{T}}$ is the vector of random residual effects.

The expectations were $\mathrm{E}\left(\mathrm{y}_{\mathrm{T}}\right)=\mathrm{X}_{\mathrm{T}} \mathrm{b}_{\mathrm{T}}, \mathrm{E}\left(\mathrm{s}_{\mathrm{T}}\right)=0$, $\mathrm{E}\left(\mathrm{mgs}_{\mathrm{T}}\right)=0, \mathrm{E}\left(\mathrm{pe}_{2}\right)=0, \mathrm{E}\left(\mathrm{e}_{\mathrm{T}}\right)=0 ; \mathbf{s}_{\mathrm{T}}$ and $\mathbf{m g s}_{\mathrm{T}}$ were assumed to follow a multivariate normal distribution with mean 0 and covariance matrix $\mathbf{G} \otimes \mathbf{A}$, where $\mathbf{G}$ is a $4 \times 4$ direct-maternal variances-covariance matrix as shown below:

$$
\mathbf{G}=\left[\begin{array}{cccc}
\sigma_{s 1}^{2} & \sigma_{s 1, s 2} & \sigma_{s 1, m g s 1} & \sigma_{s 1, m g s 2} \\
\sigma_{s 1, s 2} & \sigma_{s 2}^{2} & \sigma_{s 2, m g s 1} & \sigma_{s 2, m g s 2} \\
\sigma_{s 1, m g s 1} & \sigma_{s 2, m g s 1} & \sigma_{m g s 1}^{2} & \sigma_{m g s 1, m g s 2} \\
\sigma_{s 1, m g s 2} & \sigma_{s 2, m g s 2} & \sigma_{m g s 1, m g s 2} & \sigma_{m g s 2}^{2}
\end{array}\right]
$$

and $\mathbf{A}$ is the additive genetic relationship among sire and maternal grandsires and all recorded ancestors. The variances were calculated as $\operatorname{Var}\left(\mathbf{p e}_{2}\right)=\mathrm{PE} \otimes \mathbf{I}_{\mathrm{c}}$ and $\operatorname{Var}(\mathbf{e})=\mathbf{R} \otimes \mathbf{I}_{e}$, where $\mathbf{R}$ is a diagonal matrix of size 2 for heifers and cows; $\mathbf{I}_{c}$ is the identity matrix of permanent environmental effects of cows; $\mathbf{I}_{\mathrm{e}}$ is the identity of each record, and $\otimes$ is the Kronecker matrix product. The A matrix was constructed using all the available pedigree information on all animals, which was traced back to the 1950s. Animals with unknown parents were grouped based on their birth year, country of origin, and sex according to standard DataGene genetic evaluation procedures.

The direct additive genetic variance $\left(\sigma_{D}^{2}\right)$, the maternal additive genetic variance $\left(\sigma_{M}^{2}\right)$, and the covariance between direct and maternal effect $\left(\sigma_{D, M}\right), \mathrm{h}^{2}$, and genetic correlation between direct and maternal genetic effects $\left(r_{D, M}\right)$ were derived as described by Steinbock et al. (2003) from the variances and covariances matrix of sire and sire-maternal grandsire models shown above or directly from the animal model variance-covariance matrices:

$$
\begin{gathered}
\sigma_{D}^{2}=4 \sigma_{\text {sire }}^{2}, \\
\sigma_{M}^{2}=4 \sigma_{m g s}^{2}+\sigma_{\text {sire }}^{2}-4 \sigma_{\text {sire }, m g s},
\end{gathered}
$$

and

$$
\sigma_{D, M}=4 \sigma_{\text {sire }, m g s}-2 \sigma_{\text {sire }}^{2}
$$

The genetic correlation between the direct and maternal effects was calculated as

$$
r_{D, M}=\frac{\sigma_{D, M}}{\sqrt{\sigma_{D}^{2} \sigma_{M}^{2}}} .
$$

The phenotypic variance $(\mathrm{P})$ for heifers was

$$
\sigma_{P}^{2}=\sigma_{\text {sire }}^{2}+\sigma_{m g s}^{2}+\sigma_{e}^{2} .
$$

The phenotypic variance $(\mathrm{P})$ for cows was

$$
\sigma_{P}^{2}=\sigma_{\text {sire }}^{2}+\sigma_{m g s}^{2}+\sigma_{p e}^{2}+\sigma_{e}^{2} .
$$

Using the phenotypic variance, the direct and maternal $\mathrm{h}^{2}$ were calculated as

$$
h_{D}^{2}=\frac{\sigma_{D}^{2}}{\sigma_{P}^{2}}
$$

and

$$
h_{M}^{2}=\frac{\sigma_{M}^{2}}{\sigma_{P}^{2}} .
$$

Genetic parameters were also estimated assuming that heifer GL is the same trait as cow GL. Thus, model 1 and calculation of heritabilities simplified to a single trait repeatability model. Genetic parameters were also estimated ignoring maternal effects, where the phenotypic variance simplifies to $\sigma_{P}^{2}=\sigma_{D}^{2}+\sigma_{p e}^{2}+\sigma_{e}^{2}$.

\section{Genetic Evaluations}

Calculation of GL EBV Using All Data. Genetic evaluations of direct and maternal GL were performed using the genetic parameters estimated from the animal model that included both the direct (animal that was born) and maternal (the cow that calved) effects. For practical reasons and given the parameters, GL in heifers and cows were assumed to be the same trait, so parameters from a single repeatability model were used to calculate EBV:

$$
\mathbf{y}=\mathbf{X b}+\mathbf{Z a}+\mathbf{W m}+\mathbf{C p e}+\mathbf{e} .
$$

The definitions of all fixed effects in model 2 are the same as in model 1 but considered GL of heifers and cows as 
the same trait; $\mathbf{Z}$ is incidence matrix for random effects of the calf born (direct), a is vector of random genetic effect of calf, $\mathbf{W}$ is the incidence matrix for random calving cow genetic effect (maternal), $\mathbf{m}$ is the vector of random maternal genetic effects of the cow, $\mathbf{C}$ is the incidence matrix for random permanent environmental cow effect fitted to account for repeated records, pe is the vector for permanent random environmental effect of cow, and $\mathbf{e}$ is the vector of residual errors. In the case of animal model, phenotypic variance was:

$$
\sigma_{P}^{2}=\sigma_{A}^{2}+\sigma_{M}^{2}+\sigma_{p e}^{2}+\sigma_{e}^{2} .
$$

The main aim of calculating EBV for direct and maternal effects was to illustrate the genetic trend for GL for direct and maternal genetic effects. Genetic trends were calculated based on EBV of animals with at least 50\% reliability. The current interest in Australia (and elsewhere) is in direct EBV for GL, so genetic evaluations ignoring maternal genetic effects were also calculated. From the model that ignored the maternal effect, DTD and DBV were calculated and used for testing genomic prediction.

Calculation of GL EBV Using the Early Data. The GL EBV were predicted using GL data that was available up to 2012 (early data). The EBV from these data were used (1) to assess the predictive ability of parent average and direct genomic value (DGV) of validation bulls and (2) to assess if direct GL EBV of bulls affects the CInt of cows that were mated to these bulls in the validation data. The early data were also used to calculate DTD, TD, and DBV, which were used as response variables for genomic prediction.

The model used to calculate DTD, TD, and DBV was a single-trait repeatability animal model ignoring the maternal genetic effect. The TD were calculated from raw phenotype data by fitting a model that included the fixed herd-year-season-age of calving (classes), the age of cow (covariate), and the random permanent environment effect the dam of the animal. The DTD for bulls were then calculated from the TD of their daughters after correcting for the genetic merit of their mate according to DataGene procedures describe elsewhere (e.g., Nieuwhof et al., 2010). The DBV were calculated as described in Jairath et al. (1998).

\section{Genomic Analysis and Cross Validation}

Genotype data of about 64,000 animals were provided by DataGene. Of these, 13,016 animals with GL DTD, TD, or DBV were retained for our study. The animals were genotyped with a variety of commercially available SNP chips, with the majority being genotyped using the Illumina Bovine SNP50 Bead Chip (Illumina Inc., San Diego, CA). The marker data were edited by DataGene following the protocols described by Hayes et al. (2009) and Nieuwhof et al. (2010) so that 46,726 SNP markers were retained. These genotypes were used to construct a genomic relationship matrix (GRM) following Yang et al. (2010).

Using the GRM, the following model was used for genomic prediction

$$
\mathbf{y}=1 \mu+(\mathbf{X b})+\mathbf{Z g}+\mathbf{e},
$$

where $\mathbf{y}$ is a vector of response variables (DTD or TD or DBV), $\mu$ is the mean, $\mathbf{b}$ is a vector of the effect of the type of the response variable (TD for individual animals or DTD for bulls), $\mathbf{X}$ is a design matrix relating $\mathbf{y}$ to the type of the response variable, $\mathbf{Z}$ is a design matrix relating records to DGV of animals, $g$ is a vector of $\mathrm{DGV} \sim N\left(0, \mathbf{G} \sigma_{g}^{2}\right)$, where $\mathbf{G}$ is the GRM, $\sigma_{g}^{2}$ is the genomic variance, and $\mathbf{e}$ is a vector of random residuals, $\sim N\left(0, \mathbf{D} \sigma_{e}^{2}\right)$, where $\sigma_{e}^{2}$ is residual variance and $\mathbf{D}$ is a diagonal matrix derived from Garrick et al. (2009) to account for the reliability of the response variable.

Genomic predictions were performed considering 3 sets of animals in the reference population, as shown in Table 2); (1) using DTD or DBV of 3,220 bulls with at least 10 progeny with GL in the early data; (2) using DTD of 3,529 bulls with at least 5 progeny with GL records or using DBV of 3,801 bulls with EBV reliability of $\geq 40 \%$ in early data; and (3) using DTD of all bulls in 2 and TD of 7,817 cows with $\geq 2$ GL records in early data or DBV of all bulls in 2 and animals with EBV reliability of $\geq 40 \%$ in early data. The DBV were used instead of DTD or TD for genomic prediction to assess if the reliability of genomic prediction can be improved by using DBV as response variable instead of DTD or $\mathrm{TD}$, as is done in several Interbull-member countries (Interbull, 2018).

The reliability of genomic predictions using different reference sets were compared with the reliability of parent average based on 451 validation bulls with only parent average in the data before 2013 (early data), but with at least 10 or more progeny in the 2016 data (all data). The mean and standard deviation of DTD and DBV of the validation bulls based on 2016 data (all data) is shown in Table 2. The reliability of DGV were ascertained for the validation bulls by calculating (1) the coefficient of determination between response variable and the corresponding DGV in the validation animals $\left(\mathbf{R}^{2}\right.$; the proportion of variance in DTD or DBV explained by DGV) and (2) the slope of the regression (b) of DTD or DBV on DGV. When calculating $\mathrm{R}^{2}$ and 
Table 2. Number animals and mean and SD of response variables [daughter trait deviation (DTD) or trait deviation (TD) and deregressed breeding value (DBV)] of animals used as reference and validation set for gestation length (GL)

\begin{tabular}{lrcc}
\hline & & \multicolumn{2}{c}{ Response variable } \\
\cline { 3 - 4 } Category & Number & Mean DTD & Mean DBV \\
\hline Bulls $\geq 10$ progeny & 3,220 & $5.07(3.12)^{1}$ & $4.73(2.44)^{1}$ \\
Bulls $\geq 5$ progeny & 3,529 & $5.05(3.24)^{1}$ & - \\
Bulls with EBV reliablity $\geq 40 \%$ & 3,801 & - & $5.05(2.48)^{1}$ \\
Bulls $\geq 5$ progeny and cows & 11,202 & $4.46(3.21)^{1}$ & - \\
All animals with EBV $\geq 40 \%^{3}$ & 11,712 & & $4.95(3.08)^{1}$ \\
Validation set (bulls only) & 451 & $4.69(3.00)^{1}$ & $4.73(2.44)^{1}$ \\
\hline
\end{tabular}

${ }^{1}$ Standard deviations in parentheses.

${ }^{2}$ DTD of 3,529 bulls with $\geq 5$ progeny and TD of 7,817 cows with $\geq 2$ GL records.

${ }^{3} \mathrm{DBV}$ of 3,801 bulls and 7,911 cows with EBV reliability of $\geq 40 \%$.

the slope (b), the number of effective daughters per bull was used as weight in ASReml (Gilmour et al., 2009).

Adjusted $\mathrm{R}^{2}\left[(\mathrm{DGV} \text {, true breeding value })^{2}\right]$ of validation bulls were calculated by dividing the $\mathrm{R}^{2}(\mathrm{DGV}$, DTD, or DBV) by the average reliability of the DTD or DBV (calculated from the $h^{2}$ and the number of effective daughters) of validation bulls. These were compared with the theoretical or expected reliabilities (e.g., Hayes et al., 2009) that were calculated for the ith bull as $1-P E V_{i} / \sigma_{g}^{2}$, where $P E V_{i}$ is the prediction error variance of the DGV for the $i$ th bull and $\sigma_{g}^{2}$ is the genomic variance. Here, the prediction error variance was assumed to be the square of the error associated with DGV of the ith bull.

\section{Effectiveness of GL EBV of Bulls}

Bulls with direct GL EBV based on data up to 2012 that were mated to cows after 2012 were used to assess the effect of GL EBV on CInt of their mates. The following fixed effect model was used to estimate the effect of GL EBV of bulls on CInt of their mates:

$$
\begin{gathered}
\mathrm{y}_{\mathrm{ijklm}}=\mu+\mathrm{P}_{\mathrm{i}}+\mathrm{M}_{\mathrm{j}}+\mathrm{HYS}_{\mathrm{k}}+\operatorname{lin}(\mathrm{GL} \mathrm{EBV}) \\
+\operatorname{lin}(\text { CalvingTo1stS })+\operatorname{pol}(\text { Age }, 2)+\mathrm{PE}_{1}+\mathrm{e}_{\mathrm{ijklm}},
\end{gathered}
$$

where $y_{\mathrm{ijklm}}$ is the response variable, which in this case is the CInt of the cows that are mated to bulls with GL
$\mathrm{EBV} ; \mu$ is the mean; $\mathrm{P}_{\mathrm{i}}$ the parity effect; $\mathrm{M}_{\mathrm{j}}$ is month of calving; $\mathrm{HYS}_{\mathrm{k}}$ is the effect of herd-year-season-age of calving; lin(GL EBV) is the linear regression on GL EBV of the bull; lin(CalvingTo1stS) is the linear regression on the interval from calving to first service in days of the cow; pol(Age,2) is linear and quadratic regression on age at calving of the cow; $\mathrm{PE}_{1}$ is the random permanent environmental effect of the cow; and $\mathrm{e}_{\mathrm{ijkl}}$ is the random error term. The same analyses were also performed by categorizing the bulls into 3 groups based on their GL EBV (short, average, and long). The data used for these analyze are shown in Table 3. The effect of the bulls' GL EBV on the GL of their mates was also estimated using cows that had valid GL data (Table 3). The model for this analysis is same as model 4 except that it did not include the regression of the interval from calving to the first service.

\section{RESULTS}

\section{Mean GL and Genetic Parameter Estimation}

Male calves represented $21 \%$ of the animals with valid GL data and were carried for $1.2 \mathrm{~d}$ longer than female calves, which represented $35 \%$ of the animals. Animals whose sex was not known represented $45 \%$ of the calves with valid GL data and were carried for $0.2 \mathrm{~d}$ longer than female calves. Table 1 shows that the mean GL of cows was about 1.5 d longer than heifers. Table

Table 3. Description of the direct gestation length (GL) EBV of bulls and calving interval (CInt) data of cows (their mates) used for validating the effectiveness of GL EBV of bulls

\begin{tabular}{lcccc}
\hline GL EBV category of bulls & No. of bulls & Mean GL EBV (SD) & No. of CInt records & Mean CInt (SD) \\
\hline Short GL EBV & 155 & $-3.88(1.16)$ & 8,195 & $376.8(46.1)$ \\
Medium GL EBV & 727 & $-0.08(1.28)$ & 35,078 & $378.6(45.9)$ \\
Long GL EBV & 114 & $3.63(1.04)$ & 4,715 & $380.1(45.8)$ \\
Total & 996 & $-0.24(2.31)^{1}$ & 47,988 & $378.4(45.9)^{1}$ \\
\hline
\end{tabular}

${ }^{1}$ Mean GL EBV (of bulls) or mean CInt (of cows) and SD of all animals used for these analyses. 
Table 4. Genetic parameters $\left(\mathrm{h}^{2}\right.$ on the diagonal and correlation between heifer and cow traits above diagonal) for gestation length (GL) estimated assuming sire-maternal grandsire model in first (Heifer) and second and later parities (Cow) as 2 traits

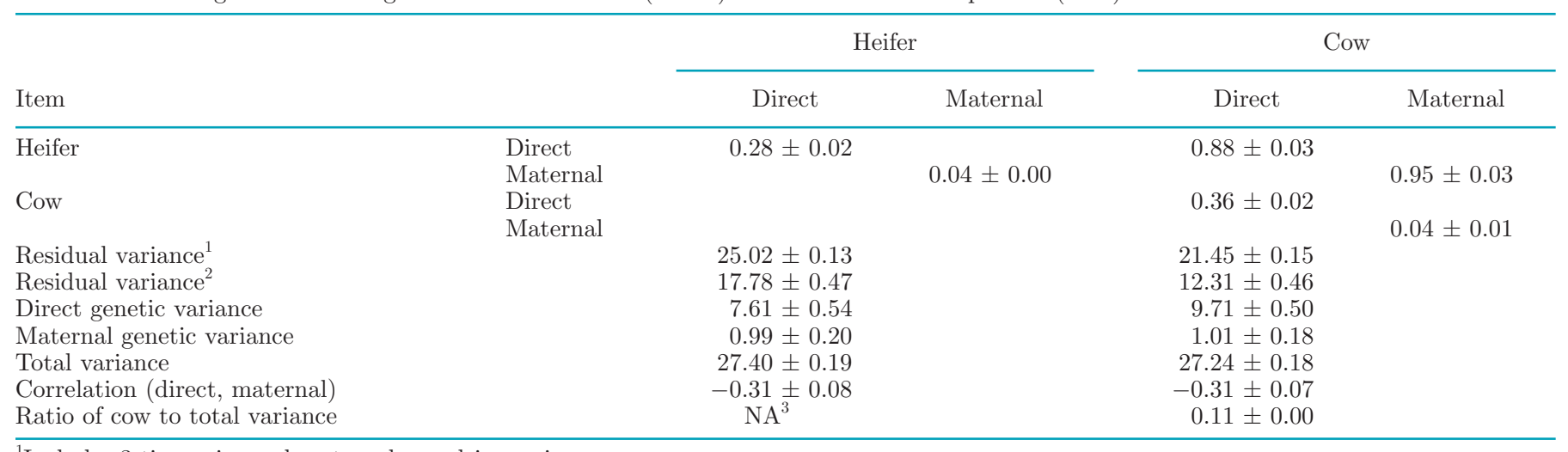

${ }^{1}$ Includes 3 times sire and maternal grandsire variance.

${ }^{2}$ After accounting for 3 times sire and maternal grandsire variance.

${ }^{3} \mathrm{NA}=$ not applicable, because heifers have only 1 record.

1 also shows the variability in cow GL was smaller than in heifers. The direct $\mathrm{h}^{2}$ of GL was slightly higher $(0.36)$ in cows than in heifers $(0.28)$, which was largely due to the smaller residual variance in cows (Table 4). On the other hand, the maternal $\mathrm{h}^{2}$ in both heifers and cows was the same. The direct and maternal genetic correlation between GL in heifers and cows was reasonably high, suggesting that they could be considered as the same trait (Table 4). The genetic correlation between direct and maternal effects was negative and varied from -0.31 (Table 4; heifer versus cow data from sirematernal grandsire model) to -0.45 (Table 5) when heifer and cow data were analyzed jointly in a repeatability sire-maternal grandsire or animal model. The ratio of the permanent environmental variance to the total variance is small and the maternal repeatability (sum of maternal $\mathrm{h}^{2}$ and the ratio of permanent environmental to the total variance) was also small (about

Table 5. Genetic parameters for gestation length (GL) estimated assuming sire-maternal grandsire and animal repeatability model using all parity data

\begin{tabular}{lcr}
\hline Item & $\begin{array}{c}\text { Sire-maternal } \\
\text { grandsire model }\end{array}$ & \multicolumn{1}{c}{$\begin{array}{c}\text { Animal } \\
\text { model }\end{array}$} \\
\hline${\text { Direct } \mathrm{h}^{2}}^{2}$ & $0.29 \pm 0.01$ & $0.29 \pm 0.03$ \\
Maternal h$^{2}$ & $0.03 \pm 0.01$ & $0.03 \pm 0.01$ \\
Residual variance $^{1}$ & $21.80 \pm 0.17$ & $19.41 \pm 0.54$ \\
Residual variance $^{2}$ & $12.62 \pm 0.59$ & $19.41 \pm 0.54$ \\
Direct genetic variance & $7.89 \pm 0.82$ & $8.45 \pm 0.84$ \\
Maternal genetic variance & $0.80 \pm 0.27$ & $0.74 \pm 0.24$ \\
Total variance & $27.20 \pm 0.42$ & $29.27 \pm 0.54$ \\
Correlation (direct, maternal) & $-0.45 \pm 0.11$ & $-0.36 \pm 0.12$ \\
Ratio of cow to total variance & $0.09 \pm 0.01$ & $0.02 \pm 0.01$ \\
\hline
\end{tabular}

${ }^{1}$ Includes 3 times sire and maternal grandsire variance.

${ }^{2}$ After accounting for 3 times sire and maternal grandsire variance.
$0.05)$ when animal model was used and 0.11 when sirematernal grandsire model was used.

\section{Genetic Evaluations}

The distribution of direct GL EBV of bulls shows an opportunity to select for this component of the trait. The mean EBV, in addition to the standard deviation for direct and maternal GL for bulls and cows, are shown in Table 6. As expected, the ranges are slightly wider in bulls than in cows for both direct and maternal GL. The correlation between direct and maternal EBV are negative $(-0.55$ in bulls and -0.66 in cows).

Figure 1 illustrates the direct genetic and maternal genetic trend for GL. The variability in maternal genetic trend for GL was less than direct GL. The direct GL genetic trend in bulls rose between 1991 and 1995 and was somewhat stable until 2005 in bulls and 2006 in cows. Starting in 2005 in bulls and in 2006 in cows, there appears to be some reduction in direct GL EBV that coincided with the introduction of the genetic evaluation for fertility in 2003 in Australia. In bulls born from 2005 to 2011, the direct GL EBV has reduced by $0.19 \mathrm{~d} / \mathrm{yr}$. Overall, the trend in cows is less clear than in bulls for both component of GL.

\section{Genomic Prediction and Validation}

Table 7 shows the $\mathrm{R}^{2}$ (squared correlation between DGV and the response variable), regression, and adjusted reliability and expected reliability for direct GL in 451 validation bulls when using DTD and DBV as response variables. Relative to parent average, the use of the most reliable bulls (i.e., 3,220) as the reference set increased the $\mathrm{R}^{2}$ from 0.17 to 0.25 when DTD was used 
Table 6. Mean, minimum (min), and maximum (max) direct and maternal EBV for gestation length (GL) from a model where both direct and maternal effects are fitted ${ }^{1}$

\begin{tabular}{|c|c|c|c|c|c|c|c|}
\hline \multirow[b]{2}{*}{ Sex } & \multirow[b]{2}{*}{ No. } & \multicolumn{3}{|c|}{ Direct GL } & \multicolumn{3}{|c|}{ Maternal GL } \\
\hline & & Mean & Min & $\operatorname{Max}$ & Mean & Min & $\operatorname{Max}$ \\
\hline Bulls & 9,921 & $0.69(2.35)$ & -12.45 & 11.67 & $0.29(0.63)$ & -4.33 & 4.71 \\
\hline
\end{tabular}

${ }^{1}$ Cows have a minimum of 5 GL records and bulls with at least 5 progeny or grand progeny records; values in parenthesis are standard deviations of EBV.

as response variable and 0.22 to 0.26 when $\mathrm{DBV}$ was used as response variable (Table 7). If the same number of bulls are used when either the DBV or DTD is used as a response variable then similar $\mathrm{R}^{2}$ are obtained, which was expected because the correlation between DTD and DBV for validation bulls is close to 1 (0.98).

The addition of cows to the reference set increased the $\mathrm{R}^{2}$ by a further 0.03 when DTD (for bulls) and TD (for cows) were used and by 0.04 when DBV (for both bulls and cows) was used (Table 7). Having TD for cows and DTD for bulls, as well as the use of DBV of all animals with EBV reliability of at least $40 \%$ in the reference set increased $R^{2}$, adjusted $R^{2}$, and the expected reliability (Table 7). In fact, using DBV for all animals when the reliability of GL EBV was at least $40 \%$ increased the $\mathrm{R}^{2}$ by 0.02 compared with the use of DTD and TD. This was largely the result of an increased number of animals in the reference set (Table 7). On the other

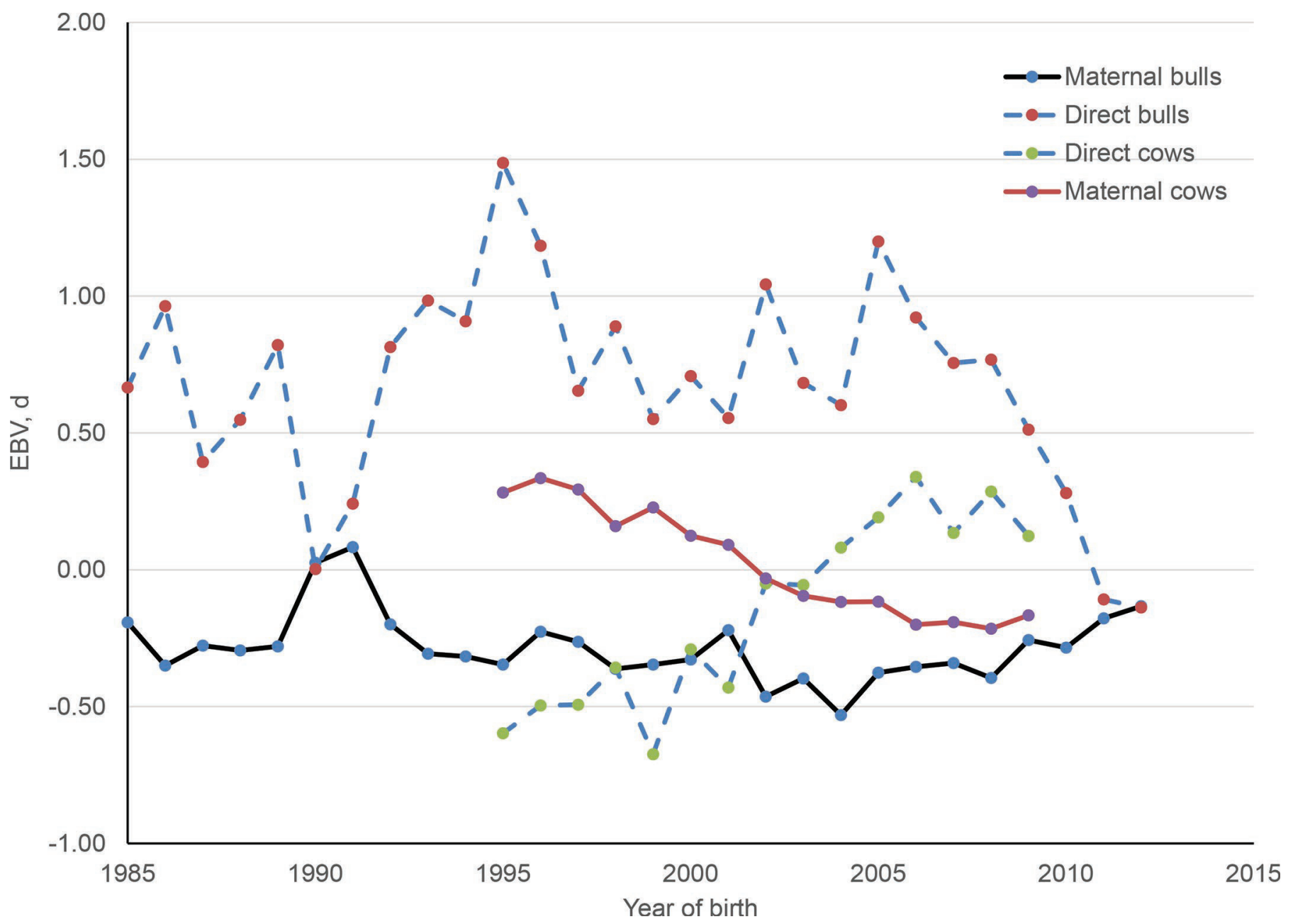

Figure 1. Genetic trend for direct and maternal EBV for cows and bulls for gestation length of Holstein breed. 
Table 7. Reliability of prediction [expressed as $\mathrm{R}^{2}$, direct genomic values (DGV) or parent average, and daughter trait deviations (DTD)] and regression (b) of DTD on DGV in 451 validation bulls and adjusted $\mathrm{R}^{2}$ (Adj. $\mathrm{R}^{2}$ ) and expected reliability (Exp. Rel.) for gestation length (GL) using different group reference animals

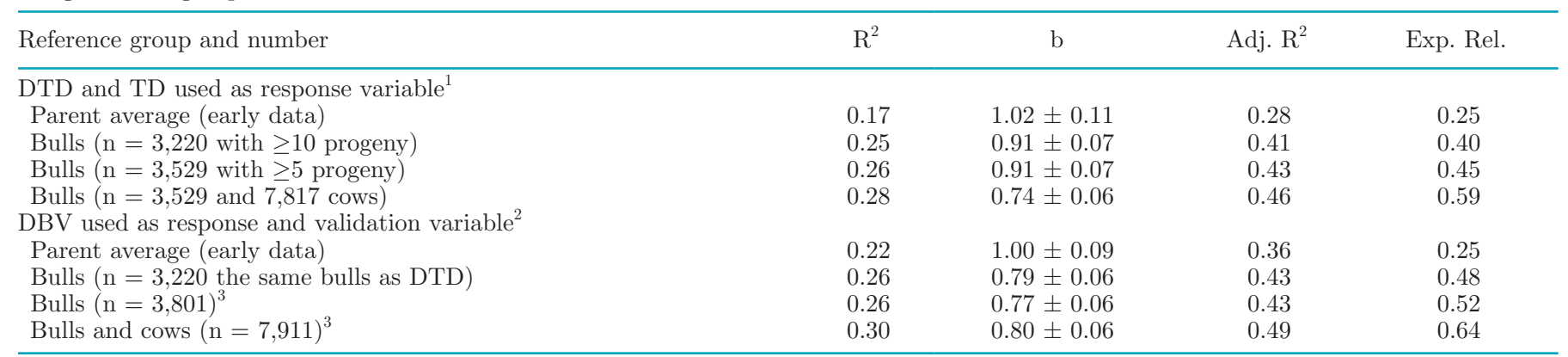

${ }^{1}$ DTD of bulls and TD of cows used as response variable but DTD of bulls used for validation.

${ }^{2}$ Deregressed breeding value (DBV) is used instead of DTD to calculate $\mathrm{R}^{2}, \mathrm{~b}, \mathrm{Adj} . \mathrm{R}^{2}$, and expected reliability.

${ }^{3}$ Bulls or bulls and cows with EBV reliability of $\geq 0.40$ used in the reference set.

hand, increasing the number of animals in the reference set by including all animals with TD decreased the $\mathrm{R}^{2}$ and increased bias (results not tabulated) compared with those in Table 7.

In general, the addition of animals with TD into the reference set as well as the use of DBV as response variable suggests that genomic prediction is likely to overestimate BV of validation animals, as can be seen from the regression coefficient b in Table 7 . In contrast, parent average predicted DTD or DBV with no bias (Table 7). The regression coefficient values in Table 7 show that use of DTD of bulls with at least 5 progeny is slightly below 1.0 but is not significantly different from 1.0. The genomic cross-validation results show that adjusting the reliability of DGV of animals, by dividing the $\mathrm{R}^{2}$ by the average reliability of the DTD or DBV, will give reliabilities of genomic BV of 0.4 to 0.5 (Table 7).

\section{Effectiveness of GL EBV of Bulls}

Table 8 shows the effect of direct GL EBV of bulls on the CInt and GL of cows that were mated to them. The use of short GL EBV bulls is expected to decrease the CInt of their mates by $2.1 \mathrm{~d}$ relative to the use of long-GL EBV bulls (Table 8). Table 8 also shows a larger effect on GL of cows than on CInt. The regression analyses show that a 1-d increase in GL EBV of

Table 8. Effect of direct GL EBV of bulls on the calving interval (CInt) and gestation length (GL) of their mates as the deviation from that of the short-GL EBV bulls

\begin{tabular}{lcc}
\hline Category of GL EBV & CInt of mates & GL of mates \\
\hline Medium GL EBV & $0.86 \pm 0.54$ & $1.45 \pm 0.14$ \\
Long GL EBV & $2.09 \pm 0.81$ & $3.53 \pm 0.21$ \\
\hline
\end{tabular}

bulls that are mated to cows is expected to increase CInt by $0.16 \pm 0.06 \mathrm{~d}(P \approx 0.04)$ and her GL by 0.44 $\pm 0.02 \mathrm{~d}(P \approx 0.001)$.

\section{DISCUSSION}

Genetic parameters needed for routine genetic evaluations of GL were estimated for Holstein cattle using data collected over 20 yr from Australian herds. The key objective in having routine genetic evaluations of GL is to help manage calving patterns by selectively mating short-GL EBV bulls to cows that have not become pregnant toward the end of the mating season. The results from our study, in agreement with Norman et al. (2011) and Winkelman and Spelman (2001), show that the choice of bulls can be used to decrease the GL of cows to which they are mated. Interestingly, the effect of selective mating of cows to short direct GL EBV bulls is expected to marginally reduce the CInt of cows. As more than $60 \%$ of Australian herds are predominantly pasture-based (Dairy Australia, 2018a) and, consequently, tight calving patterns are essential for the success of the system, the availability of GL EBV will be useful to manage calving patterns and minimize the need for calving induction.

Several models for estimating genetic parameters and genetic evaluation of GL were tested. As observed in both New Zealand (Winkelman and Spelman, 2001) and the United States (CDCB, 2017), direct GL EBV can be used for practical management decisions to reduce the GL of mates, which is especially useful for systems reliant on pasture growth.

The correlation between direct EBV from heifers and cows is high and, given that the amount of data on heifers is limited, the model of choice is a repeatability animal model that uses both heifer and cow GL data. Including a maternal genetic effect in the model en- 
abled us to calculate maternal EBV, which was useful to know the extent of variation (Table 6) and to see if any unfavorable trend existed in this component of the trait (Figure 1). However, currently the reliability and usefulness of this EBV is low. The correlation of direct GL EBV from a model that either accounted for or ignored maternal effects are very high (close to 1.0 in bulls with more than 5 progeny and above 0.96 in all animals), which suggests that the simpler model is sufficient for genetic evaluation of GL.

The mean GL of both heifer and adult cows in the current study (Table 1) were slightly longer than those in US Holsteins (Norman et al., 2009), reported to be 278 and $279 \mathrm{~d}$ in heifers and cows, respectively, but are close to those in New Zealand Holstein, which are about $282 \mathrm{~d}$ (Winkelman and Spelman, 2001). Jamrozik et al. (2005) reported GL of $280 \mathrm{~d}$ for both heifers and cows in Canadian Holstein.

The direct and maternal $\mathrm{h}^{2}$ estimated in the current study were lower than most literature estimates (Winkelman and Spelman, 2001; Hansen et al., 2004; Norman et al., 2009); they were also lower than estimates from an earlier Australian study by McClintock (2004), who reported a direct $h^{2}$ of 0.51 and 0.48 in heifers and adult cows and a maternal $\mathrm{h}^{2}$ of 0.05 and 0.07 in heifers and adults, respectively. Several factors, including methodology and components, that were considered in the modeling (maternal, permanent environmental effects), as well as quality and quantity of data could have contributed to the difference between the current study and others. For example, ignoring the permanent environmental effect when estimating maternal genetic variance and could inflate the maternal $\mathrm{h}^{2}$.

The negative genetic correlations between direct and maternal genetic effects observed in the current study is consistent with several others (Cervantes et al., 2010; Jeyaruban et al., 2015; Johanson et al., 2011) and could be a mechanism to keep GL of cows within a narrow range (i.e., SD). Consequently, it is common for genetic correlations between direct and maternal effects to be negative (McClintock, 2004; Johanson et al., 2011). However, others have reported a near zero genetic correlation between direct and maternal effects in cattle (Hansen et al., 2004; Eaglen et al., 2013).

The range in direct GL EBV of bulls in the current study is similar to the United States, where a standard deviation of $2.8 \mathrm{~d}$ was reported for Holstein bulls (CDCB, 2017). The direct GL EBV calculated in our study was positively correlated (0.24) with the EBV for CInt, but the correlation of EBV for maternal GL with CInt EBV is near zero ( 0.04). Eaglen et al. (2013) reported a genetic correlation of 0.19 between direct GL and CInt, which is close to our estimate, and a negative correlation of maternal GL $(-0.29)$ with
CInt. Amer et al. (2016) reported a stronger genetic correlation between female fertility traits and direct GL compared with the genetic correlation of female fertility traits with maternal GL, which agrees with our results.

In both the New Zealand (LIC, 2018) and the United States (Wright and VanRaden, 2017), the genetic trend in direct GL EBV has been declining, as in Australia. The declining genetic trend in the United States of direct GL EBV is related to selection for improved calving ease, fertility, yield, and productive life (Wright and VanRaden, 2017), whereas in New Zealand it could be largely due to selection for short GL. The declining genetic trend in direct GL EBV of bulls and cows in Australia coincides with the decline in CInt EBV of bulls starting in 2005 observed by Haile-Mariam et al. (2013), who used similar data. The decline in GL EBV observed could partly be due to use of bulls from countries where selection for short GL is practiced. Comparison of direct GL EBV of bulls from different Interbull-member countries based on Australian data shows variability that can be utilized; as expected, bulls from New Zealand have one of the shortest mean GL EBV (results not tabulated). On the other hand, the rise in GL EBV of bulls we observed between 1991 and 1995 (Figure 1) may be associated with the decline in fertility observed in Australian Holstein cattle in the same period (Haile-Mariam et al., 2013).

The reliability of DGV for direct GL of young animals with no phenotype data observed in the current study were lower than the $65 \%$ reliability observed in the United States (CDCB, 2017), reflecting the difference in the size of the reference populations between the 2 countries. The expected reliability for GL EBV of animals with genotype-only data that will be published by DataGene when bull and cow data are used in the reference set calculated are similar to those for several other traits in Australia and are also close to those in the United States (CDCB, 2017).

The effectiveness of GL EBV of bulls on the GL and CInt of their mates observed in the current study is similar with that observed in the United States (Norman et al., 2011) and New Zealand (Jenkins et al., 2016). Even if the benefit of using short-GL bulls to mate to heifers or cows on their GL and CInt is initially small, its effect on overall fertility performance can be significant because cows that calve early in a season are also likely to be present for mating earlier in subsequent seasons, which means the likelihood of calving within the calving season is successively increased (e.g., Jenkins et al., 2016). That means the benefit of even a small difference in EBV may prove to be beneficial in seasonal calving system. However, as female fertility is particularly important in production systems where pasture growth is matched to milk production, other 
genetic and herd-management strategies, such as better overall reproduction management and the use of bulls with high female fertility breeding value, are crucial for maintaining a 365-d CInt while also reducing the need for calving induction. The Australian Dairy Industry (Dairy Australia, 2018b) has plans to gradually phase out calving induction as a means to address animal welfare issues, and the strategic use of short-GL EBV bulls can contribute to phasing out of calving induction.

At this stage, GL EBV in Australia is to be used as management tool, particularly in herds that practice pasture-based production systems, to complement other efforts to improve fertility. This is similar to the approach in the United States (CDCB, 2017). Even in the pasture-based dairy production system in New Zealand, where farmers had GL EBV for over $20 \mathrm{yr}$, it is still not included in the economic index. Despite this, there are claims that GL of cows has decreased by 1.3 d over a 10-yr period (LIC, 2018). That GL EBV are to be used as a management tools means the gain may not be cumulative, but it is worth noting that the benefit of using short GL EBV bulls is immediate. Based on Australia Holstein cows born between 2003 and 2013, the genetic trend in CInt EBV was $-0.25 \mathrm{~d} /$ yr (our unpublished Australian data), suggesting that the reduction in CInt because of using short-GL EBV bulls is approximately equivalent to up to 8 yr ( 4 to 8 yr) selection on fertility EBV. Similarly, farmers can reduce the use of long-GL EBV bulls (e.g., bulls with above average GL EBV), which may have additional benefit of reducing calving difficulty (e.g., Wright and VanRaden, 2017).

\section{CONCLUSIONS}

Data collected on GL from Australian dairy herds were used to calculate genetic parameters and EBV for GL. The genetic parameter estimates and EBV were similar to those observed in the literature. The result showed that direct GL is the most heritable and useful trait for predominantly pasture-based dairy production systems where a tighter calving pattern is required. The observed variation in the direct GL EBV of bulls means that selective use of bulls can aid in managing calving patterns or to help in predicting calving dates, and possibly help in phasing out calving induction, which is practiced in some pasture-based seasonal or split calving herds in Australia. The use of short-GL bulls can decrease the CInt and GL of their mates by 2.1 and $3.5 \mathrm{~d}$, respectively, compared with long-GL EBV bulls. The use of EBV on direct GL to identify bulls that have shorter GL may be useful in production systems where calving occurs over a short time (e.g., seasonal or split calving systems of Australia).
Current selection practices for improved female fertility appear to have resulted in some decline in the trend of direct GL EBV of bulls starting in 2005. Therefore, in addition to selective use of short-GL bulls to mate cows that do not become pregnant early in the mating season, better reproductive management, and the use of high female fertility bulls remain crucial for the success of a pasture-based dairy production system. On average, for animals with genotype data BV of their GL can be predicted with about 50 to $60 \%$ expected reliability depending the response variable and the size of reference set.

\section{ACKNOWLEDGMENTS}

We thank DataGene Ltd. (Melbourne, Australia) for provision of data and Phil Bowman (Agriculture Victoria) for extraction of the data. We also thank Gert Nieuwhof of DataGene for constructive discussion and providing the genotype data. This research was funded by DairyBio, which is a co-investment of Dairy Australia with Agriculture Victoria (Melbourne).

\section{REFERENCES}

Amer, P. R., K. Stachowicz, G. M. Jenkins, and S. Meier. 2016. Short communication: Estimates of genetic parameters for dairy fertility in New Zealand. J. Dairy Sci. 99:8227-8230.

Cervantes, I., J. P. Gutiérrez, I. Fernändez, and F. Goyache. 2010. Genetic relationship among calving ease, gestation length and survival to weaning in the Asturiana de los Valles beef cattle breeds. J. Anim. Sci. 88:96-101.

Council on Dairy Cattle Breeding (CDCB). 2017. Gestation length. Accessed March 2018. https://queries.uscdcb.com/reference/ CDCB_Reference_Sheet_Gestation_Length_08_07_2017_Final .pdf.

Dairy Australia. 2018a. Cows and farms. Accessed March 2018. https:/ /www.dairyaustralia.com.au/industry/farm-facts/cows-and-farms.

Dairy Australia. 2018b. Cow welfare. Accessed April 2018. https:// www.dairyaustralia.com.au/farm/animal-management/animal -welfare/cow-welfare.

Eaglen, S. A. E., M. P. Coffey, J. A. Woolliams, and E. Wall. 2013. Direct and maternal genetic relationships between calving ease, gestation length, milk production, fertility, type, and lifespan of Holstein-Friesian primiparous cows. J. Dairy Sci. 96:4015-4025.

Garrick, D. J., J. F. Taylor, and R. L. Fernando. 2009. Deregressing estimated breeding values and weighting information for genomic regression analyses. Genet. Sel. Evol. 41:55.

Gilmour, A. R., B. J. Gogel, B. R. Cullis, and R. Thompson. 2009 ASReml User Guide Release 3.0. VSN International Ltd., Hemel Hempstead, UK.

Haile-Mariam, M., P. J. Bowman, and J. E. Pryce. 2013. Genetic analyses of fertility and predictor traits in Holstein herds with low and high mean calving intervals and in Jersey herds. J. Dairy Sci. 96:655-667.

Hansen, M., M. S. Lund, J. Pedersen, and L. G. Christensen. 2004. Gestation length in Danish Holsteins has weak genetic associations with stillbirth, calving difficulty, and calf size. Livest. Prod. Sci. 91:23-33.

Hayes, B. J., P. J. Bowman, A. C. Chamberlain, K. Verbyla, and M. E. Goddard. 2009. Accuracy of genomic breeding values in multibreed dairy cattle populations. Genet. Sel. Evol. 41:51. 
Interbull. 2018. National genomic evaluation. Accessed April 2018. http://www.interbull.org/ib/interbull.

Jairath, L., J. C. M. Dekkers, L. R. Schaeffer, Z. Liu, E. B. Burnside, and B. Kolstad. 1998. Genetic evaluation for herd life in Canada. J. Dairy Sci. 81:550-562.

Jamrozik, J., J. Fatehi, G. J. Kistemaker, and L. R. Schaeffer. 2005. Estimates of genetic parameters for Canadian Holstein female reproduction traits. J. Dairy Sci. 88:2199-2208.

Jenkins, G. M., P. Amer, K. Stachowicz, and S. Meier. 2016. Phenotypic associations between gestation length and production, fertility, survival, and calf traits. J. Dairy Sci. 99:418-426.

Jeyaruban, M. G., D. J. Johnston, B. Tier, and H.-U. Graser. 2015. Genetic parameters for calving difficulty using complex genetic models in five beef breeds in Australia. Anim. Prod. Sci. 56:927933.

Johanson, J. M., P. J. Berger, S. Tsuruta, and I. Misztal. 2011. A Bayesian threshold-linear model evaluation of perinatal mortality, dystocia, birth weight, and gestation length in a Holstein herd. J. Dairy Sci. 94:450-460.

Livestock Improvement Corporation (LIC). 2018. Products-and-services/artificial-breeding/short-gestation-length-semen. Accessed June 2018. https://www.lic.co.nz/products-and-services/artificial -breeding/short-gestation-length-semen/.

McClintock, S. 2004. Genetic evaluation of dystocia in Australian Holstein-Friesian cattle, PhD Dissertation. Institute of Land and Food Resources, The University of Melbourne, Melbourne, Australia.
Nieuwhof, G. J., K. T. Beard, K. V. Konstantinov, P. J. Bowman, and B. J. Hayes. 2010. Implementation of genomics in Australia. Interbull Bull. 42:35-39.

Norman, H. D., J. R. Wright, M. T. Kuhn, S. M. Hubbard, J. B. Cole, and P. M. VanRaden. 2009. Genetic and environmental factors that affect gestation length in dairy cattle. J. Dairy Sci. 92:22592269 .

Norman, H. D., J. R. Wright, and R. H. Miller. 2011. Potential consequences of selection to change gestation length on performance of Holstein cows. J. Dairy Sci. 94:1005-1010.

Steinbock, L., A. Näsholm, B. Berglund, K. Johansson, and J. Philipsson. 2003. Genetic effects on stillbirth and calving difficulty in Swedish Holsteins at first and second calving. J. Dairy Sci. $86: 2228-2235$

Winkelman, A. M., and R. J. Spelman. 2001. Selection for reduced gestation length in New Zealand dairy cattle. Proc. Assoc. Adv. Anim. Breed. Genet. 14:63-66.

Wright, J.R., and P.M. VanRaden. 2017. Genetic evaluation of gestation length as a trait of the service sire. J. Dairy. Sci. 100(Suppl. 2):42. (Abstr.)

Yang, J., B. Benyamin, B. McEvoy, S. Gordon, A. Henders, D. Nyholt, P. Madden, A. Heath, N. Martin, G. Montgomery, M. E. Goddard, and P. M. Visscher. 2010. Common SNPs explain a large proportion of the heritability for human height. Nat. Genet. 42:565-569. 